\title{
Study on Debris Movement in EDM Gap
}

\author{
Masanori KUNIEDA ${ }^{*}$ and Kenji YANATORI* \\ (Received June 27, 1996)
}

\begin{abstract}
* Department of Mechanical Systems Engineering
Tokyo University of Agriculture \& Technology

Koganei, Tokyo 184, Japan
\end{abstract}

\begin{abstract}
In this paper, we describe the calculation of debris particle motion caused by the electrostatic force in narrow gaps filled with dielectric liquid in EDM processes. The debris particle moves towards to one electrode and returns to the other repeatedly due to electrophoresis. The distribution of electric potential in the gap is computed using the finite-difference method, and then the electrostatic force applied to the particle is calculated. The equation of motion for the particle motion is solved and the results obtained are compared with experimental results obtained using an image converter camera.
\end{abstract}

Key words: electric discharge machining, debris particle, electrophoresis, discharge gap

\section{INTRODUCTION}

To obtain an electric discharge in clean oil the gap length must be less than several $\mu \mathrm{m}$. In electrical discharge machining (EDM) processes, however, since the dielectric liquid is contaminated with debris particles, the gap length is much longer than that for an uncontaminated dielectric liquid. Kunieda and $\mathrm{Mori}^{1}{ }^{1}$ investigated the quantitative relationship between the gap length and the volumetric density of debris particles in EDM discharge gaps. They found that the gap length changes from 20 to $100 \mu \mathrm{m}$ depending on the gap flushing conditions when a peak pulse current of $21 \mathrm{~A}$ and a pulse duration of $250 \mu_{\mathrm{s}}$ are used.

The simplest explanation for the decrease in the dielectric breakdown strength is that the electrically conductive debris particles reduce the apparent gap length by a distance equal to the diameter of the particles. However, the measured gap length is occasionally longer than $100 \mu \mathrm{m}$, whereas the measured average diameter of debris particles is only $25 \mu \mathrm{m}^{1)}$. This result can be explained in terms of the movement of debris particles in the gap, which was observed by Suda and Sata $^{2)}$ and Mori and Kunieda ${ }^{3)}$. They found that the debris particles move between the anode and cathode in a direction perpendicular to the electrode surfaces due to electrophoresis. They also observed that some particles are linked in series to form chains parallel to the electric field.

From the observed results Mori and Kunieda $^{3)}$ hypothesized that in the gap there are numerous chains of particles which almost bridge the gap and that discharge occurs at the site at which the end of a chain is closest to the opposite electrode surface. Bommeli et al. ${ }^{4}$ statistically investigated the distribution of discharge delay time to demonstrate that discharge occurs after an activation time which is necessary for debris particles to form bridges between the electrodes.

From the point of view of the machining accuracy of EDM processes, it is likewise very important to analyze the electrophoresis-induced motion of debris particles. This is because the machining accuracy is affected by the gap length distribution and determination of the gap length is closely related to the formation of particle bridges. Calculation of the particle velocity due to electrophoresis will give an indication of the time required for particles to form bridges and thus of the discharge delay time. Furthermore, measurement of the leakage current during the discharge delay time will give an indication of the number of debris particles in the gap because each charged particle carries a certain electric charge which is determined by the electric field and the particle diameter.

In the present work we calculate the electrophoresis-induced motion of a particle and obtain the particle velocity and the electric charge carried by a particle. The calculated results are compared with the experimental results obtained using a high speed image converter camera.

\section{CALCUlATION OF ELECTROSTATIC FORCE}

\subsection{Calculation Model}

Figure 1 shows the dimensions of the particle and the gap used in the analysis. Here we assume a period during the discharge delay time at which the open voltage is being applied but discharge has not yet occurred. The particle, which is spherical and made of steel, is moving in the dielectric liquid in a direction perpendicular to the plane parallel electrodes. In this situation we can apply an axisymmetrical calculation model. The diameter of the particle is $30 \mu \mathrm{m}$ and the gap length is 120 


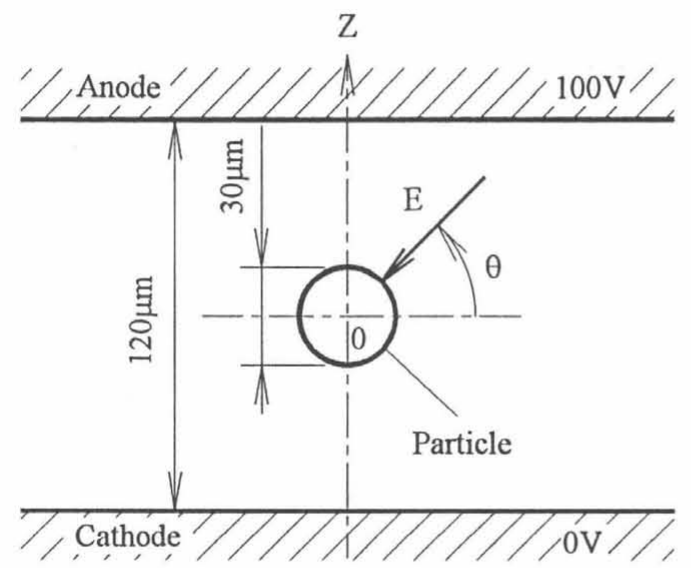

Fig.1 Calculation model

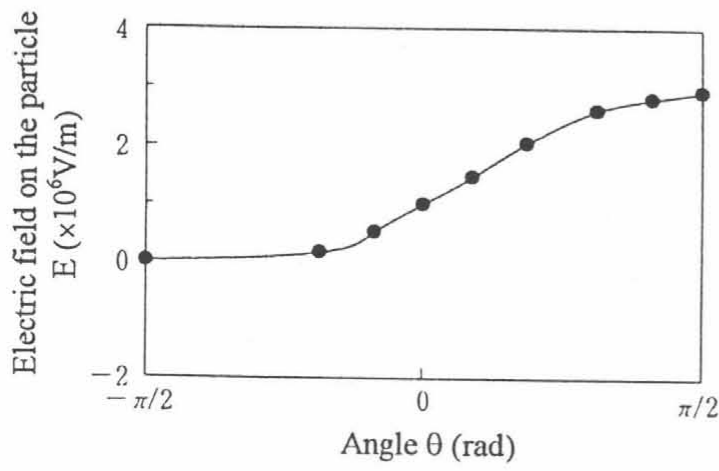

Fig.3 Electric field on the particle. (Particle position: $-45 \mu \mathrm{m}$, particle diameter: $30 \mu \mathrm{m})$

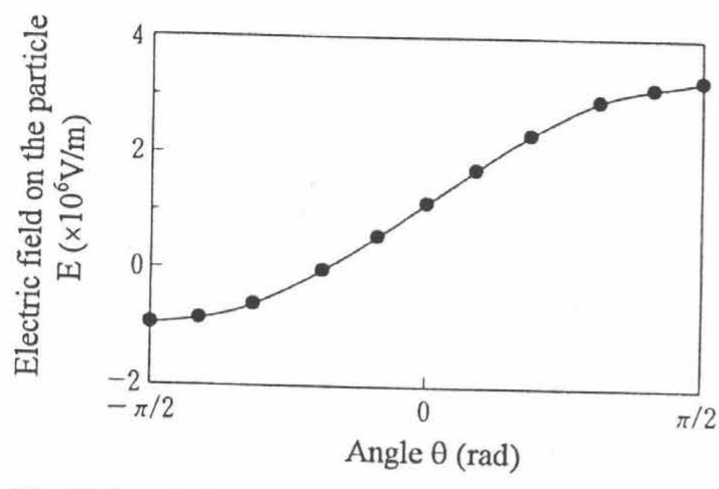

Fig.5 Electric field on the particle. (Particle position: $0 \mu \mathrm{m}$, particle diameter: $30 \mu \mathrm{m})$

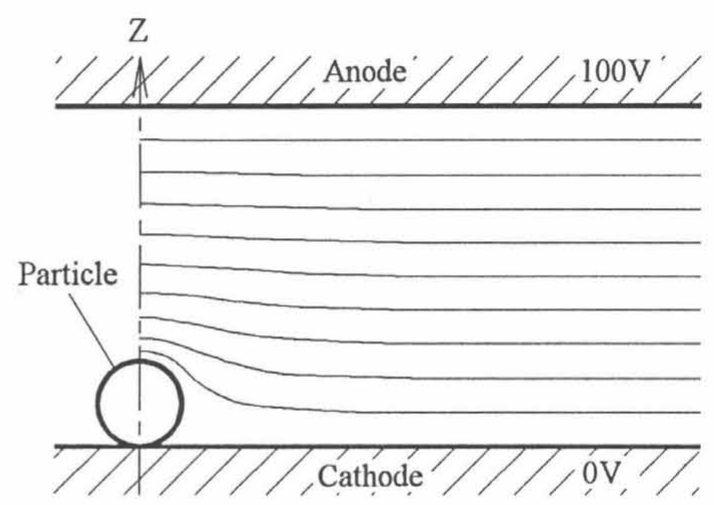

Fig.2 Equipotential lines in the gap. (Particle position: $-45 \mu \mathrm{m}$ )

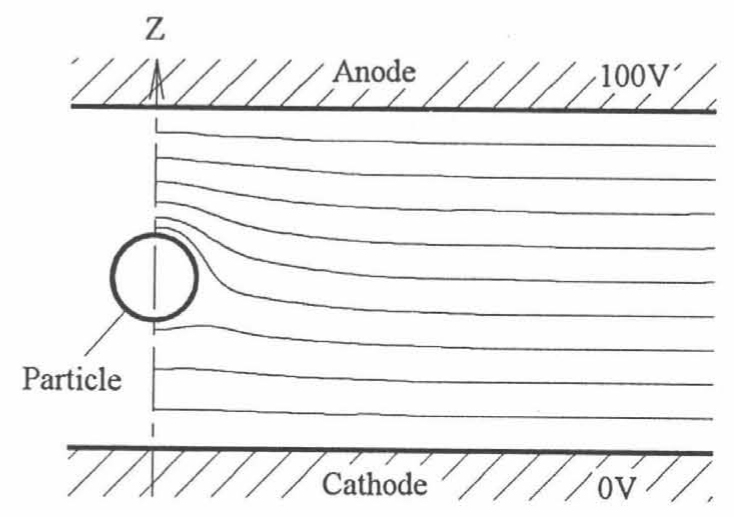

Fig.4 Equipotential lines in the gap. (Particle position: $0 \mu \mathrm{m})$

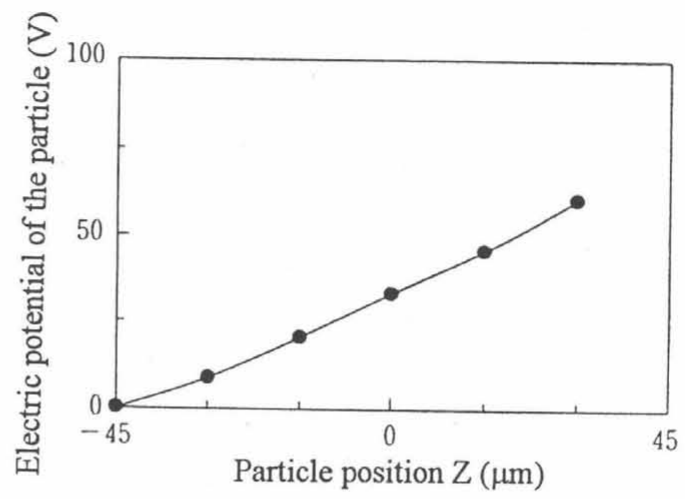

Fig.6 Relationship between particle position and electric potential. (Particle diameter: $30 \mu \mathrm{m}$ ) 
$\mu \mathrm{m}$, which are typical values under medium pulse energy conditions. The potential difference between the electrodes is $100 \mathrm{~V}$ during the discharge delay time. The origin of the $z$-axis is set at the center of the gap.

\subsection{Calculation of Electrostatic Force}

When a sphere of conductive material is placed in an electric field, a charge is induced on the surface of the sphere. The surface charge density $\sigma$ can be expressed as

$$
\sigma=-\varepsilon E,
$$

where $E$ is the electric field strength on the surface and $\varepsilon$ is the permittivity of the dielectric liquid. Here $E$ is considered to be positive when the electric field is directed towards the center of the sphere. The force per unit area, $f$, is given by

$$
f=\frac{\sigma^{2}}{2 \varepsilon}=\frac{\varepsilon E^{2}}{2} \text {. }
$$

Hence the electrostatic force applied to the sphere along the $z$-axis can be obtained by integrating the $z$-component of $f$ over the surface of the sphere as follows:

$$
F_{e}=\int_{-\frac{\pi}{2}}^{\frac{\pi}{2}} \sin \theta \frac{\varepsilon E^{2}}{2}(2 \pi r \cos \theta) r d \theta,
$$

where $r$ is the radius of the sphere.

\subsection{Calculation of Electric Field}

To compute the electrostatic force using Eq. (3), the electric field distribution should be determined. We first consider the case in which the sphere is in contact and equipotential with the cathode surface. The electric potential distribution is computed using the finite-difference method with the boundary condition that the electric potential of the sphere is equal to that of the cathode. The result is shown in Fig. 2. Then the electric field strength at the surface of the sphere is calculated from the gradient of the electric potential. Figure 3 shows the calculated electric field strength as a function of the angle $\theta$, which is defined in Fig. 1.

Next we consider the case in which the sphere is detached from the cathode and located in the gap. In this case, the electric potential of the sphere is unknown, but the total charge transferred to the sphere when it was in contact with the cathode remains constant after the sphere leaves the cathode surface. Therefore, we assume an appropriate value for the electric potential of the sphere and compute the electric potential distribution in the gap in order to calculate the electric field on the sphere. Then the total charge on the sphere $Q$ can be obtained by integrating the surface charge density over the surface of the sphere.

$$
Q=\int_{-\frac{\pi}{2}}^{\frac{\pi}{2}} \sigma(2 \pi r \cos \theta) r d \theta
$$

where $\sigma$ is given by Eq. (1). If the calculated result does not agree with the charge calculated when the sphere was in contact with the cathode, the assumed value of the electric potential is corrected and the above procedure is repeated until the value of $Q$ becomes equal to that when the sphere is in contact with the cathode. Figures 4 and 5 show the obtained electric potential distribution in the gap and the electric field strength at the surface of the sphere, respectively, when the sphere is at the center of the gap moving towards the anode. Figure 6 shows the calculated change in the electric potential of the sphere as it approaches the anode.

\subsection{Calculated Results of Electrostatic Force}

Figure 7 shows the calculated change in the electrostatic force applied to the sphere $F_{e}$ when the sphere is moving towards the anode. It is found that the electrostatic force increases as the sphere approaches the anode. Here $F_{e}[\mu \mathrm{N}]$ can be approximated as a function of $z[\mu \mathrm{m}]$ :

$$
\begin{aligned}
F_{e}= & 0.039+8.56 \times 10^{-5} z \\
& +2.16 \times 10^{-6} z^{2}+6.10 \times 10^{-8} z^{3} \\
& +2.12 \times 10^{-10} z^{4}+1.83 \times 10^{-11} z^{5} .
\end{aligned}
$$

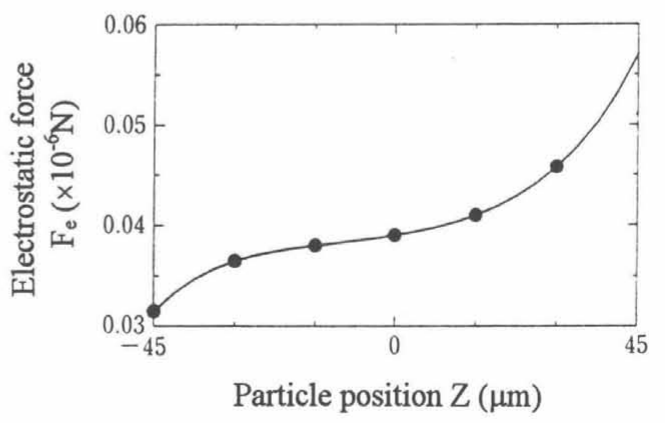

Fig.7 Relationship between particle position and electrostatic force. (Particle diameter: $30 \mu \mathrm{m}$, gap distance: $120 \mu \mathrm{m}$, gap voltage: $100 \mathrm{~V}$ ) 


\section{SOLUTION OF EQUATION OF MOTION}

\subsection{Equation of Motion}

Considering gravity and the drag force, the equation of motion for the sphere is written as

$$
m \frac{d^{2} z}{d t^{2}}=F_{e}-F_{v}-m g,
$$

where $F_{v}$ is the drag force, $m$ is the mass of the sphere and $g$ is the acceleration due to gravity. The drag force, which is composed of friction drag and form drag is expressed as ${ }^{5}$

$$
F_{v}=\frac{\rho \operatorname{cs}\left(\frac{d z}{d t}\right)^{2}}{2} .
$$

Here $\rho$ is the density of the dielectric liquid, $s$ is the projection of the sphere onto a surface perpendicular to the movement of the sphere, and $c$ is the drag coefficient, which is defined as

$$
c=\frac{10}{\sqrt{R_{e}}},
$$

where $R_{c}$ is the Reynolds number.

\subsection{Calculated Motion in the first path}

Using calculation software (Mathematica), the equation of motion was solved under the initial conditions that the sphere is in contact with the cathode $(z=-45)$ and its velocity is zero $(d z / d t=0)$ at $t=0$. Figure 8 shows the results calculated for the first path of sphere motion from departure from the cathode to the first collision with the anode. The results obtained when the effect of gravity or that of the drag force is ignored are shown in the same graph. It is found that the effect of gravity is small but that the drag force is significant. When

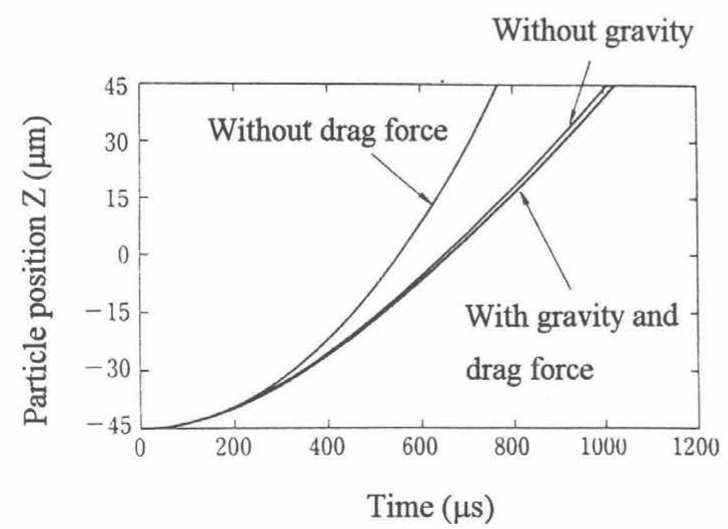

Fig. 8 Calculated movement of the particle in the gap. (Particle diameter: $30 \mu \mathrm{m}$, gap distance: $120 \mu \mathrm{m}$, gap voltage: $100 \mathrm{~V}$ ) both effects are taken into account, the traveling time for the first path is $1030 \mu \mathrm{s}$, the sphere velocity when the first collision occurs is $0.138 \mathrm{~m} / \mathrm{s}$, and the average velocity for the first path is 0.088 $\mathrm{m} / \mathrm{s}$.

\subsection{Calculation of Reciprocating Motion}

When the sphere comes into contact with the anode surface, the negative charge is discharged and the sphere is charged again, this time with positive charge. Then the electrostatic force which acts in the $-z$ direction accelerates the sphere back towards the cathode. In this way, the sphere moves back and forth between the electrodes. Eventually, the reciprocating motion reaches a steady state because the electrostatic force is balanced by the drag force as the velocity increases. When gravity is neglected, the motion is symmetrical in both directions in the steady state. We concluded that the motion reached a steady state when the rate of change of the collision velocity for each path became less than $0.01 \%$.

The calculated results shown in Fig. 9 show how the reciprocating motion reaches a steady state when the coefficient of restitution is 1.0. It was found that the steady state is reached after two reciprocating cycles. In the steady state, the time taken to travel between the electrodes is $660 \mu \mathrm{s}$ and the average velocity is $0.136 \mathrm{~m} / \mathrm{s}$.

Figure 10 shows the results calculated for a coefficient of restitution 0.5 . In this case, the steady state is reached after one reciprocating cycle, the time taken to travel between the electrodes is $810 \mu \mathrm{s}$ and the average velocity is $0.111 \mathrm{~m} / \mathrm{s}$ in the steady state.

\subsection{Electric current carried by one sphere}

The total electric charge on one sphere $Q$ can be calculated using Eq. (4) assuming that the sphere

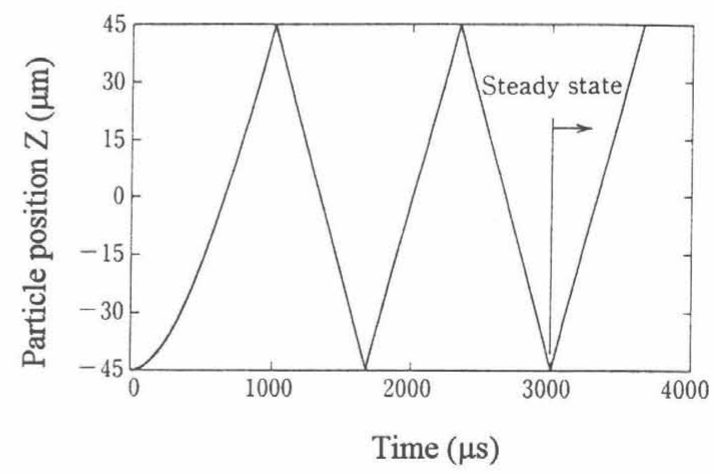

Fig.9 Transient movement of the particle after application of gap voltage. (Coefficient of restitution: 1, particle diameter: $30 \mu \mathrm{m}$, gap distance: $120 \mu \mathrm{m}$, gap voltage: $100 \mathrm{~V}$ ) 


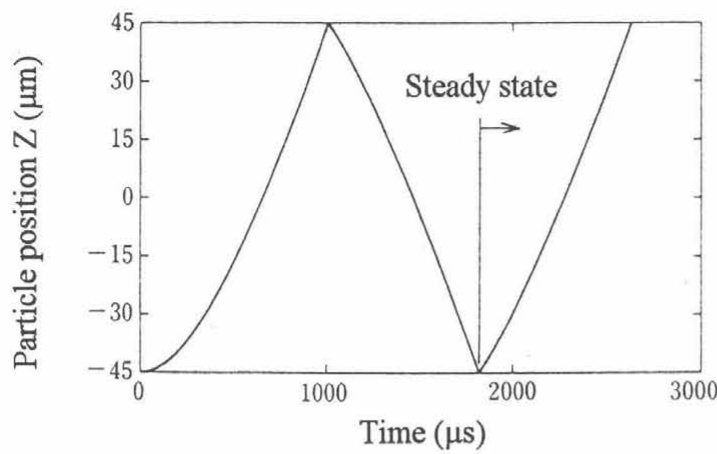

Fig.10 Transient movement of the particle after application of gap voltage. (Coefficient of restitution: 0.5 , particle diameter: $30 \mu \mathrm{m}$, gap distance: $120 \mu \mathrm{m}$, gap voltage: $100 \mathrm{~V}$ )

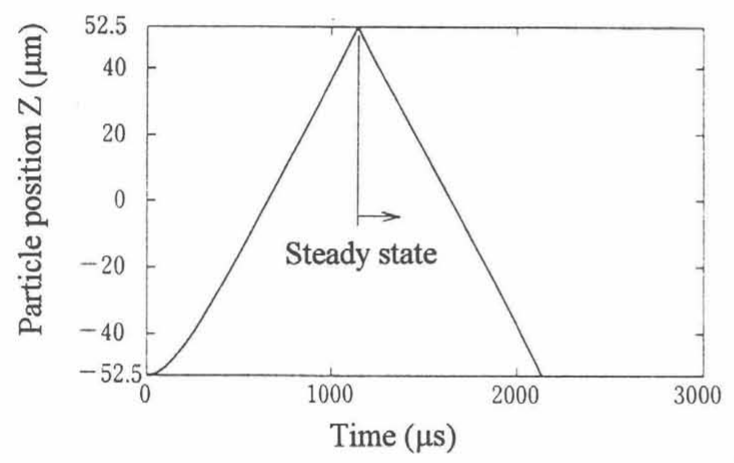

Fig.12 Transient movement of the particle after application of gap voltage. (Coefficient of restitution: 1 , particle diameter: $15 \mu \mathrm{m}$, gap distance: $120 \mu \mathrm{m}$, gap voltage: $100 \mathrm{~V}$ )

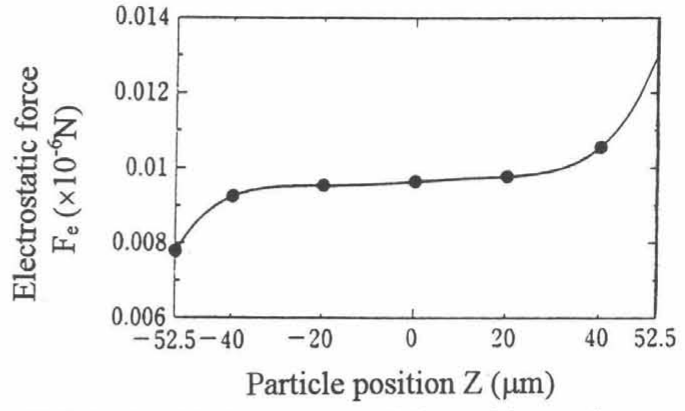

Fig.11 Relationship between particle position and electrostatic force. (Particle diameter: $15 \mu \mathrm{m}$, gap distance: $120 \mu \mathrm{m}$, gap voltage: $100 \mathrm{~V}$ )

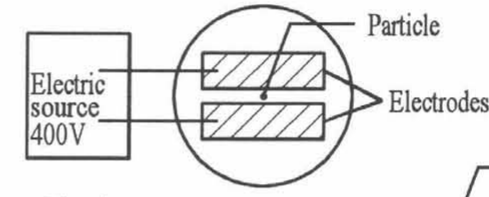

Optical

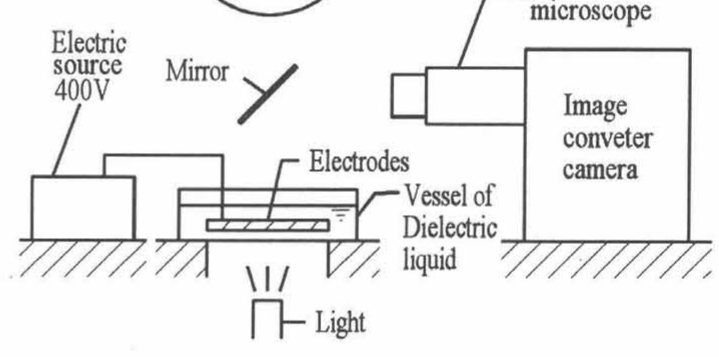

Fig.13 Measuring system

Table 1 Influence of particle diameter on its movement

\begin{tabular}{|c|c|c|c|c|c|}
\hline \multirow{2}{*}{$\begin{array}{c}\text { Particle } \\
\text { diameter } \\
(\mu \mathrm{m})\end{array}$} & \multirow{2}{*}{$\begin{array}{l}\text { Charge } \\
\text { (C) }\end{array}$} & \multicolumn{2}{|c|}{ For the first path } & \multicolumn{2}{|c|}{ Steady state } \\
\hline & & $\begin{array}{l}\text { Traveling time } \\
\qquad(\mu \mathrm{s})\end{array}$ & $\begin{array}{l}\text { Average velocity } \\
(\mathrm{m} / \mathrm{s})\end{array}$ & $\begin{array}{l}\text { Traveling time } \\
\qquad(\mu \mathrm{s})\end{array}$ & $\begin{array}{l}\text { Average velocity } \\
(\mathrm{m} / \mathrm{s})\end{array}$ \\
\hline 30 & $-5.63 \times 10^{-14}$ & 1030 & 0.0878 & 660 & 0.136 \\
\hline 15 & $-1.40 \times 10^{-14}$ & 1140 & 0.0919 & 995 & 0.106 \\
\hline
\end{tabular}


is in contact and equipotential with either the cathode or the anode. When the relative permittivity of the dielectric liquid is 2.0 and the diameter of the sphere is $30 \mu \mathrm{m}, Q$ is calculated to be $-5.63 \times 10^{-14} \mathrm{C}$ when the sphere is in contact with the cathode. In the steady state, one sphere comes into contact with either the cathode or the anode every $1 / 660 \mu \mathrm{s}$, or 1520 times per second, and hence carries a current of $5.63 \times 10^{-14} \mathrm{C} \times$ $15201 / \mathrm{s}=8.53 \times 10^{-11} \mathrm{~A}$. It may be possible to estimate the total number of debris particles in the gap by measuring the leakage current during the discharge delay time.

\section{INFLUENCE OF SPHERE DIAMETER}

The sphere diameter was halved to $15 \mu \mathrm{m}$ keeping the gap length and gap voltage constant. The calculated electrostatic forces are shown in Fig. 11 , where the debris position $z=-52.5$ indicates that the sphere is in contact with the cathode. Compared with the case in which the sphere diameter is $30 \mu \mathrm{m}$, the electrostatic force is about one fourth at $z=0$. Figure 12 shows the reciprocating motion of the sphere which departs from the cathode with an initial velocity of $0 \mathrm{~m} / \mathrm{s}$ when the coefficient of restitution is 1.0. Table 1 shows the total charge, the traveling time for one path and the average velocity for sphere diameters of 30 and $15 \mu \mathrm{m}$. The total charge is also decreased to one fourth, which is proportional to the ratio of the surface areas of the spheres. For the first path, although the average velocity of the small sphere is higher than that of the larger one, the traveling time is longer. This is because the traveling distance for one path is increased from 90 to $105 \mu \mathrm{m}$, corresponding to the decrease in diameter of $15 \mu \mathrm{m}$. In the steady state, the average velocity of the small sphere is lower than that of the large one.

\section{OBSERVATION OF MOTION}

The reciprocating motion was observed using an image converter camera. Figure 13 shows the equipments used. The frame speed was $1 \times 10^{4}$ frames per second and the exposure time was 20 $\mu \mathrm{s}$ per frame. The particle is a steel sphere with a diameter of $50 \mu \mathrm{m}$ and the gap length is 200 $\mu \mathrm{m}$. Copper electrodes were used to avoid the influence of residual magnetism in the electrodes on the steel particle. The gap voltage was $400 \mathrm{~V}$. The electrodes were horizontal to eliminate the influence of gravity. To magnify the image by a factor of 20, an optical microscope was incorporated in the image converter camera.

Figure 14 shows the measured displacement of the center of the sphere. If the motion is steady state, the coefficient of restitution can be obtained by dividing the velocity with which the sphere leaves one electrode by the velocity with which it hits the other electrode. The data in Fig. 14 were approximated using a quadratic function of time, from which it was found that the leaving velocity is $0.0945 \mathrm{~m} / \mathrm{s}$ and the hitting velocity is $0.320 \mathrm{~m} / \mathrm{s}$. Therefore, the coefficient of restitution is 0.295 .

Using the measured leaving velocity, the equation of motion was solved. The result, which is shown by the dotted line in Fig. 14, indicates that the actual motion is slower than the calculated motion. One reason for this may be that the sphere leaves the electrode surface before it has acquired $100 \%$ of the electric charge which can be obtained under the given electrostatic conditions. The rebound time is too short for the electrostatic conditions to be satisfied, and the sphere acquires a lower charge than that predicted theoretically.

Figure 15 shows the calculated electrostatic force applied to the sphere for various charge fractions. It is found that the electrostatic force is roughly proportional to the charge fraction. As shown by the solid line in Fig. 14, when it is assumed that the sphere is charged to $50 \%$ of the theoretical value, the calculated results coincide well with the measured results.

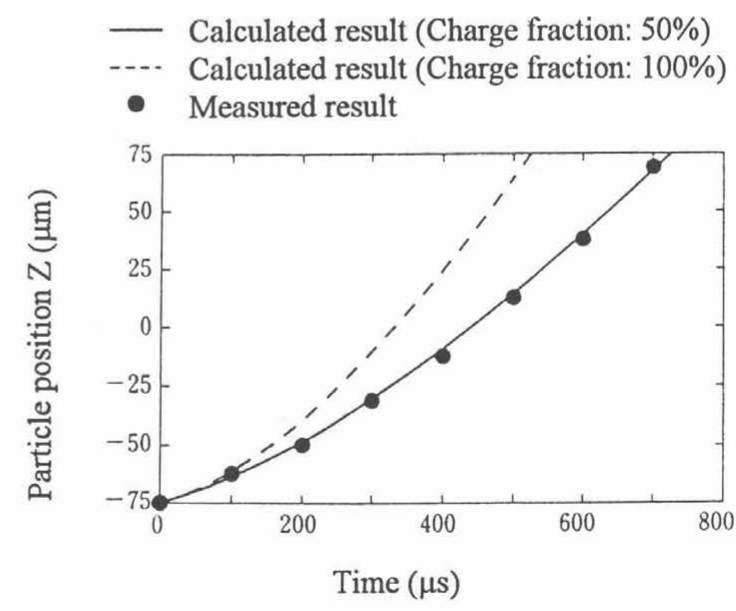

Fig.14 Comparison between measured and calculated results. (Particle diameter: $50 \mu \mathrm{m}$, gap distance : 200 $\mu \mathrm{m}$, gap voltage: $400 \mathrm{~V}$ ) 


\section{DISCUSSIONS}

In the EDM gap, the velocity of particles is so rapid that when a pulse voltage is applied, numerous chains of particles will bridge the gap in a short period and discharge will occur at the site at which each end of a chain is closest to the anode and cathode, respectively. During the discharge duration, in the vicinity of the discharge spot, chains will be broken due to the explosive expansion of a bubble of vapor from the dielectric liquid and its dissociated gases. Far from the discharge spot, however, obviously most of the chains will be sustained, and hence, when the next pulse voltage is applied, discharge will occur after an activation time which is necessary for one of the preserved chains to bridge the two electrodes. Considering the fact that the particle velocity is very high, the activation time may be extremely short, because every preserved chain is almost to complete a bridge. Consequently, it may be concluded that the activation time is the so-called discharge delay time in EDM processes.

\section{CONCLUSIONS}

The reciprocating motion of a debris particle due to electrophoresis was analyzed and the calculated results were confirmed by observation using an image converter camera. The main conclusions obtained are as follows.

(1) A debris particle which is in contact with the cathode is negatively charged and accelerated towards the anode. When the particle collides with the anode, the negative charge is discharged, the particle becomes positively charged and is accelerated towards the cathode. This model satisfactorily explains the observed results.

(2) Assuming that the coefficient of restitution is 1.0 and that the particle is fully charged, in the steady state the traveling time for one path and the average velocity of a $30-\mu \mathrm{m}-$ diameter steel particle were calculated to be $660 \mu_{\mathrm{s}}$ and $0.136 \mathrm{~m} / \mathrm{s}$, respectively.

(3) Under the same assumptions, the total charge on one particle is $5.63 \times 10^{-14} \mathrm{C}$ and the current carried by the particle is $8.53 \times 10^{-11}$ A. This result may be utilized to estimate the number of particles in the gap by measuring the leakage current during the discharge delay time.

(4) From observations, the coefficient of restitution was found to be 0.295 . Using this value, the calculated motion coincides with the observed motion when the charge fraction is assumed to be $50 \%$.
(5) The discharge delay time is considered to be the activation time which is necessary for debris particles to form bridges between the anode and cathode.

\section{REFERENCES}

1) M. Kunieda, M. Mori: Relation between Debris Concentration and Discharge Gap Length in EDM Process, Proc. of Asian Electrical-machining Symposium '95, pp.1-6.

2) T. Suda, T. Sata: Movement of Electrically Conductive Particles in the Gap of EDM Process, J. of JSEME, 7, 14, (1974), pp.1928 (in Japanese).

3) M. Mori, M. Kunieda: Observation of Debris Particle Movements in EDM Processes, Proc. of Spring Meeting of JSPE (1992), pp.279280 (in Japanese).

4) B. Bommeli, C. Frei, A. Ratajski: On the Influence of Mechanical Perturbation on the Breakdown of a Liquid Dielectric, J. Electrostatics, 7, (1979), pp.123-144.

5) N. P. Cheremisinoff and P. N. Cheremisinoff: Hydrodynamics of Gas-Solids Fluidization, Gulf Publishing Company (1984).

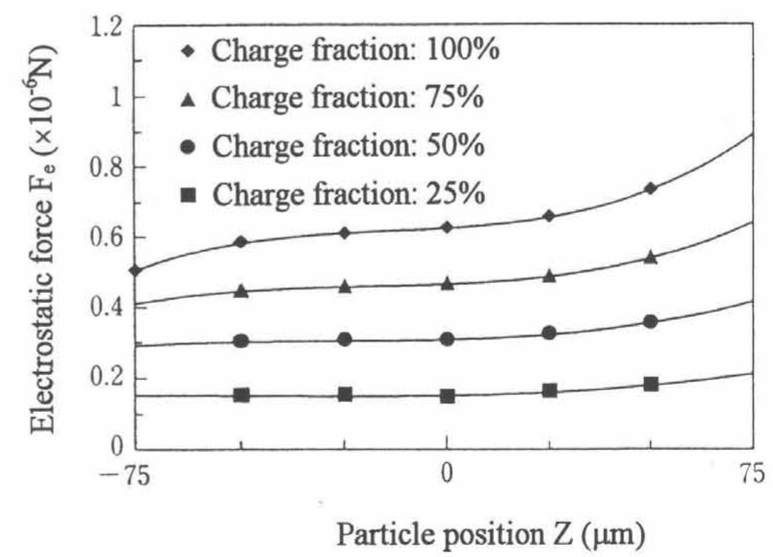

Fig.15 Influence of charge fraction on electrostatic force. 